\title{
A novel taxonomic over-view of family Vespidae of subtropical rain forest: Malakand, with three new records for Khyber Pakhtunkhwa and one for Pakistan
}

\author{
Shehzad Ahmad ${ }^{1}$, Qadeem Khan ${ }^{1}$, Qaiser Zaman ${ }^{1,2^{*}}$, Qaisar Jamal ${ }^{3}$, \\ Gauhar Rahman ${ }^{2}$, Muhammad Ismail ${ }^{1}$, Ahmad Ullah ${ }^{1}$, Roman Ali ${ }^{1}$, \\ Hamza Khan ${ }^{1}$, Umar Ali ${ }^{1}$ and Azaz Ahmad ${ }^{4}$ \\ 1. Department of Zoology, Government Postgraduate College Dargai, Malakand, Khyber Pakhtunkhwa, Pakistan \\ 2. Department of Zoology, Abdul Wali Khan University, Mardan Pakhtunkhwa, Pakistan \\ 3. Department of Zoology, University of Peshawar, Pakhtunkhwa, Pakistan \\ 4. Department of Entomology, University of Agriculture, Peshawar, Pakhtunkhwa, Pakistan \\ Corresponding author's email: zoologist.qaiser@gmail.com \\ Citation \\ Shehzad Ahmad, Qadeem Khan, Qaiser Zaman, Qaisar Jamal, Gauhar Rahman, Muhammad Ismail, Ahmad Ullah, \\ Roman Ali, Hamza Khan, Umar Ali and Azaz Ahmad. A novel taxonomic over-view of family Vespidae of \\ subtropical rain forest: Malakand, with three new records for Khyber Pakhtunkhwa and one for Pakistan. Pure and \\ Applied Biology. Vol. 11, Issue 2, pp629-638. http://dx.doi.org/10.19045/bspab.2022.110064
}

\begin{tabular}{llll}
\hline \hline Received: 19/06/2021 & Revised: 03/09/2021 & Accepted: 06/09/2021 & Online First: 18/09/2021 \\
\hline \hline
\end{tabular}

\section{Abstract}

In present study, a sum of 363 specimens were collected during August-2017 to September-2018 by visiting 14 different habitats including fields, mountains and fruit shops in Malakand, Khyber Pakhtunkhwa, Pakistan. A total of 19 species and an additional subspecies of family Vespidae were identified belonging to 10 genera and three subfamilies: Vespinae, Polistinae and Eumeninae. Out of this one genus was recorded from subfamily Vespinae, two genera were recorded from subfamily Polistinae while six genera were recorded from subfamily Eumeninae. In current study one species: Rhynchium oculatum leviscutis was recorded for the first time from Pakistan, 3 species: Rhynchium brunneum brunneum, Eumenes punctatus, Antepipona sibilans were reported for the first time from Khyber Pakhtunkhwa while 14 species were recorded from district Malakand for the first time which are: Polistes indicus, Polistes olivaceus, Polistes wattii, Ropalidia brevita, Delta dimidiatipenne, Delta esuriens, Delta pyriforme pyriforme, Ropalidia cyathiformis, Vespa orientalis, Vespa tropica, Vespa velutina, Allorhynchium metallicum, Anterhynchium abdominale abdominale, Rhynchium carnaticum, and Antepipona deflenda.

Keywords: Eumeninae; Malakand; New records; Pakistan; Polistinae; Vespidae; Vespinae Introduction

Family Vespidae belongs to order Hymenoptera, one of the 26 orders of class

Insecta. Hymenoptera comprising of more than 1, 00,000 described species and many more still to go [1]. Many species of 
Hymenoptera possess two pairs of wings, and mouthparts are modified for biting, sucking and lapping in some species. Complete metamorphosis occurs in the species of Hymenoptera [2]. Order Hymenoptera is further subdivided into two suborders; Suborder Apocrita and Suborder Symphyta. Suborder Apocrita contains five superfamilies i.e., Vespoidea, Bethyloidea, Chalcidoidea, Sphecoidea and Scolioidea [3]. Vespidae is cosmopolitan in distribution. The members of Vespidae are called wasps having about 5000 species found in tropical regions throughout the world [4]. Both social and solitary wasps are found in family Vespidae [5]. Social wasps include two subfamilies i.e., Polistinae (paper wasps) and Vespinae (hornet wasps). The solitary wasps include subfamily Eumeninae (potter wasps), Stenogastrinae, Euparagiinae and Masarinae [6]. These are predators of different kinds of insect larvae [7]. Social wasps build their nests made of paper material composed of plant material and chewed up wood materials [8]. Solitary wasps build mudhomes. Social wasps live in community that includes queen (fertile female), males and workers (sterile females). Males have thirteen antennal segments and seven abdominal segments. Whereas queen and workers have six abdominal segments and twelve antennal segments [2]. The members of Vespoidea have 11 to 13 segments in their antennae and a constriction at the base of the abdomen. The fore wings contain 6 or more cells and a sting at posterior end of the abdomen [7].

\section{Materials and Methods}

Survey was carried in District Malakand, Khyber Pakhtunkhwa, Pakistan during August-2017 to September-2018. The collection was done using hand-nets and insecticide sprays. Samples were fixed on cork-board in insect boxes using insect pins. Naphthalene balls were used as preservative. The specimens were studied using stereo microscope (Olympus Stereo SZX16, Japan).

\section{Results and Discussion}

A total of 363 specimens were collected representing three subfamilies of the family Vespidae. The subfamily Polistinae was represented by two genera: Polistes and Ropalidia; and five species: Polistes indicus, Polistes olivaceus, Polistes wattii, Ropalidia cyathiformis and Ropalidia brevita. The subfamily Vespinae contains one genus: Vespa and three species i.e., Vespa orientalis, Vespa tropica and Vespa velutina. Subfamily Eumeninae was represented by six genera: Delta, Allorhynchium, Anterhynchium, Rhynchium, Eumenes and Antepipona; and eleven species and an additional subspecies i.e., Delta dimidiatipenne, Delta esuriens, Delta pyriforme pyriforme, Allorhynchium metallicum, Anterhynchium abdominale abdominale, Anterhynchium abdominale bengalense, Rhynchium oculatum leviscutis, Rhynchium carnaticum, Rhynchium brunneum brunneum, Eumenes punctatus, Antepipona sibilans and Antepipona deflenda.

\section{Keys to the subfamilies}

Body features of all the species were compared to the keys of Das \& Gupta (1989) and Carpenter \& Nguyen (2003).

\section{Subfamily polistinae}

A total of 105 Specimens of subfamily Polistinae were collected including five species, Polistes indicus, Polistes olivaceus, Polistes wattii, Ropalidia brevita and Ropalidia cyathiformis.

Polistes indicus Stolfa, 1934 (Fig. 1)

\section{Material examined}

Haryankot $9 \hat{\sigma}(4 / 9 / 2017), 1 \hat{\sigma}(21 / 9 / 2017)$, Ghanidheri $1 \sigma^{\lambda}(15 / 9 / 2017), 1{ }^{\lambda}(4 / 10 / 2017)$.

\section{Distribution}

India, Pakistan, Iran, Iraq, Afghanistan, Oman and UAE [9-11].

\section{Remarks}

Polistes indicus has been reported from Gilgit Baltistan [12]; from Hunza, Chillas, Kohat, Abad Khel, Bahawalpur, Sindh: Ghotki, Sukkur, Allahyar Pinhwar; from Dir and Pothwar [13]; from Sindh [14]. Present study confirmed the habitats of this species for the first time from subtropical rain forest of Malakand.

Polistes olivaceus De Geer, 1773 (Fig. 2)

Material examined

$\begin{array}{lccc}\text { Ghanidheri } & 2 q & (15 / 09 / 2017), & 1 q \\ (03 / 10 / 2018), & 1 q & (06 / 05 / 2017), & 1 q\end{array}$




$\begin{array}{lccc}(11 / 06 / 2018), & 1 q & (27 / 06 / 2018), & 1 q \\ (31 / 06 / 2018), & 1 q & (12 / 07 / 2018), & 2 q \\ (13 / 08 / 2018), & 1 q & (10 / 07 / 2018), & 1 q \\ (12 / 07 / 2018), & 1 q & (15 / 07 / 2018), & 1 q \\ (11 / 08 / 2018), & 1 q & (14 / 08 / 2018), & 1 q \\ (25 / 08 / 2018), 2 q & (26 / 08 / 2018), \text { Haryankot } 2+ \\ (22 / 09 / 2017), & K h a t a k 0 s h a h 3 & 3 & (19 / 08 / 2018), \\ \text { Meherdai 5q } & (29 / 08 / 2018), & \text { Petaw } & 1+ \\ \text { (02/09/2018). } & & \end{array}$

\section{Distribution}

Bangladesh; Bhutan; Cambodia; Chagos Archipelago; Chile, Easter Is. (introduced); China (including Hong Kong and Taiwan); Egypt; Fiji; French Polynesia; Hawaii; Indonesia; Iran; Japan; Laos; Madagascar; Malaysia; Marianas; Mauritius; Myanmar; Nepal; New Caledonia; New Zealand (introduced); Oman; Pakistan; Philippines; Réunion; Samoa; Seychelles; Singapore; Society Is.; Sri Lanka; Tanzania; Thailand; Tonga; U.S.A. (introduced); Vietnam [7, 13, $15,16]$.

\section{Remarks}

Early reported from Rawalpindi and Gilgit Baltistan [17]; from Dir [13]; from Gilgit Baltistan, Punjab [18]; from region of Pothwar [13]. We reported this species for the first time from subtropical rain forest of Malakand.

Polistes wattii Cameron, 1900 (Fig. 3)

\section{Material examined}

Haryankot 1 우 (04/09/2017), Ghanidheri 1 ㅇ

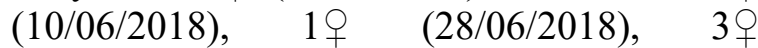
$(31 / 06 / 2018), \quad 1$ 우 $\quad(04 / 07 / 2018), \quad 1$ ㅇ $(05 / 07 / 2018), \quad 2 q \quad(06 / 07 / 2018), \quad 2$ ㅇ (09/07/2018), $\quad 1$ ㅇ $\quad(10 / 07 / 2018), \quad 13$ ㅇ (25/07/2018), 1 우 $\quad(16 / 08 / 2018), \quad 1$ 우 (18/08/2018), Dargai 1 ㅇ (03/07/2018), Khatakoshah 4 ㅇ (19/08/2018), Meherdai 1 ㅇ (29/08/2018), Petaw 1 q (02/09/2018).

\section{Distribution}

Afghanistan; China; India; Iran; Iraq; Mauritius; Oman; Pakistan; Saudi Arabia; Tajikistan; Turkmenistan; U.A.E. [7, 15, 18].

\section{Remarks}

Early reported from Rawalpindi and Attock [13]; from Gilgit Baltistan [15]; from Islamabad and Multan [17]; from Dir [14, 19]. Present study reported Polistes wattii for the first time from subtropical rain forest of Malakand.

Ropalidia brevita Das \& Gupta, 1989 (Fig. 4a, b)

\section{Material examined}

Ghanidheri $\quad 1 \sigma^{\hat{O}} \quad(15 / 09 / 2017), \quad 1$ 우 (04/06/2018), 1 우 (10/06/2018), Petaw 2ð, 9 우 (02/09/2018).

\section{Distribution}

India, Pakistan $[7,13,15]$.

\section{Remarks}

Earlier reported from Abbottabad, Ghari Habibullah, Mansehra and Islamabad [18]; from Islamabad-Rawalpindi, Attock, Murree, Jhelum and Chakwal [13]; from Rawalpindi [17]; from Sindh: Hyderabad, Mirpur Khas and Umarkot [14]. This study recorded Ropalidia brevita for the first time from subtropical rain forest of Malakand.

Ropalidia cyathiformis Fabricius, 1804 (Fig. 5)

\section{Material examined}

Haryankot 1 ㅇ (22/09/2017), Ghanidheri 3 ㅇ $(27 / 09 / 2017), \quad 2$ 운 $\quad(05 / 06 / 2018), \quad 1$ 우 (27/06/2018), $\quad 1$ 운 $\quad(01 / 07 / 2018), \quad 1+$ (03/07/2018), $\quad 2$ 우 $\quad(10 / 07 / 2018), \quad 1$ 우 $(15 / 08 / 2018), 2$ ㅇ $(16 / 08 / 2018)$, Dargai 1 우 (03/07/2018).

\section{Distribution}

Pakistan [18], Nepal, India, Sri Lanka, Burma, Java, Malaya, Sumba, Sulawesi [7], Indonesia, Vietnam, Thailand, China, Malaysia, Philippines, Myanmar [20].

\section{Remarks}

Early reported from Swat [18]; from Pothwar [13]; Rawalpindi [17]. Present report is for the first time from subtropical rain forest of Malakand.

Subfamily Vespinae

Vespa orientalis Linnaeus, 1771 (Fig. 6)

\section{Material examined}

Heroshah17우 (31/08/2017), Haryankot 1 우 (21/09/2017), Warter 1 ㅇ (14/05/2018), Ghanidheri $\quad 3$ 우 $\quad(19 / 04 / 2018), \quad 1$ ㅇ $(12 / 05 / 2018), \quad 2 q \quad(13 / 05 / 2018), \quad 2$ ㅇ $\begin{array}{llll}(01 / 06 / 2018), & 1 & (04 / 06 / 2018), & 2\end{array}$ $\begin{array}{llll}(14 / 06 / 2018), & 2 & (26 / 06 / 2018), & 1\end{array}$ $\begin{array}{llll}(31 / 06 / 2018), & 2 & (09 / 07 / 2018), & 1\end{array}$ (11/07/2018), $\quad 1$ 우 $\quad(12 / 07 / 2018), \quad 2$ 우 
$\begin{array}{llll}(15 / 07 / 2018), & 1 q & (19 / 07 / 2018), & 2 q \\ (30 / 07 / 2018), & 3 q & (02 / 08 / 2016), & 1 q \\ (04 / 08 / 2018), & 2 q & (08 / 08 / 2018), & 1 q \\ (15 / 09 / 2018), & \text { Dargai } & 2 q & (03 / 07 / 2018),\end{array}$

Khatakoshah $89(19 / 08 / 2018)$, Meherdai 5 q (29/08/2018), Petaw 2 ㅇ (02/09/2018).

\section{Distribution}

Afghanistan; Albania; Algeria; Bahrain; Bosnia \& Herzegovina; Bulgaria; China; Croatia; Cyprus; Egypt; Ethiopia; Georgia; Greece; Iran; Iraq; Israel; Italy; Jordan; Kazakhstan; Kyrgyzstan; Lebanon; Libya; Macedonia; Malta; Montenegro; Nepal; Oman; Pakistan; Romania; Russia; Saudi Arabia; Somalia; Syria; Tajikistan; Turkey; Turkmenistan; U.A.E.; Uzbekistan; Yemen [7, 15, 20].

\section{Remarks}

Early report from Punjab [7]; from Chitral, Brun and Bamboret valley [10]; from Mardan, Abbottabad and Islamabad [18]; from Attock, Rawalpindi-Islamabad, Murree, Chakwal and Jhelum [13]; from Abbottabad, Mansehra, Battagram, Havelian, Alai, Balakot and Baffa [17]. We confirmed the presence of Vespa orientalis for the first-time from subtropical rain forest of Malakand.

Vespa tropica Linnaeus, 1758 (Fig. 7)

\section{Material examined}

Heroshah 33우 (31/08/2017) Ghanidheri 1 ㅇ (06/05/2018), $\quad 1$ 우 $\quad(16 / 05 / 2018), \quad 1$ 우 $(18 / 05 / 2018), \quad 2$ 우 $\quad(30 / 05 / 2018), \quad 1$ 우 (16/08/2018), Warter 1 \% $(14 / 05 / 2018)$, Meherdai $2 q \quad(29 / 08 / 2018)$, Petaw 1 ㅇ (02/09/2018).

\section{Distribution}

Bhutan; Brunei; Cambodia; China; Indonesia; Laos; Malaysia; Myanmar; Nepal; Pakistan; Philippines; Singapore; Sri Lanka; Thailand; Vietnam $[13,15,16]$.

\section{Remarks}

Early reported from Attock, Chakwal and Rawalpindi [13]; from Islamabad, Margalla hills, Rawalakot and Rawalpindi [17]; from Peshawar, Abbottabad and Islamabad [18]; from Azad Jammu and Kashmir [14]. We reported Vespa tropica for the first time from subtropical rain forest of Malakand.

Vespa velutina Lepeletier, 1836 (Fig. 8)

\section{Material examined}

Ghanidheri $\quad 1$ 程 $\quad(02 / 05 / 2018), \quad 12$ ㅇ (11/07/2018), $\quad 1$ q $\quad(15 / 08 / 2018), \quad 1 q$ (16/08/2018), Petaw 6 ㅇ (02/09/2018).

\section{Distribution}

Afghanistan; Bhutan; China (including Taiwan); Indonesia; Laos; Malaysia;

Myanmar; Pakistan; South Korea [introduced]; Thailand; Vietnam [7, 15, 16].

\section{Remarks}

Early reported from Khyber Pakhtunkhwa [7, 10]; from Islamabad; Gilgit-Baltistan: Diamar, Afzal, Gilgit, Nomal, Chilas, Shangla, Charbagh, Swat, Garihabibullah, Shougran, Abbottabad and Nathiagali [18]; from Islamabad-Rawalpindi, Murree, Chakwal, Jhelum and Attock [13]; from Gilgit-Baltistan [15]; from Punjab, Khyber Pakhtunkhwa Islamabad and Azad Jammu and Kashmir [17]. This study reports the species of Vespa velutina for the first time from subtropical rain forest of Malakand.

\section{Subfamily Euneninae}

Delta dimidiatipenne Saussure, 1852 (Fig. 9a, b)

Material examined: Warter $2 \AA(14 / 05 / 2018)$, $10 \quad(04 / 07 / 2018), \quad$ Ghanidheri 1 \% (29/05/2018), $\quad 1$ 운 $\quad(04 / 06 / 2018), \quad 1$ 우 $(11 / 06 / 2018) \quad 1+\quad(26 / 06 / 2018), \quad 1+$ (02/07/2018), 1 ㅇ (25/08/2016).

Distribution: Afghanistan; Africa; Algeria; Arabia; Canary Islands; Chad; Djibouti; Egypt; Eritrea; Ethiopia; Iran; Madagascar; Mauritania; Nepal; Niger; Pakistan; Palestine; Spain; Somalia; South Africa; Sudan; Syria; Turkey; Uganda; Yemen [4, 15].

Remarks: Early reported from IslamabadRawalpindi, Murree, Chakwal and Jhelum [15]; from Swat [21]; from Multan, Mingora, Astore, Sukkhur and Azad Jummu and Kashmir [17]. Present study recorded Delta dimidiatipenne for the first time from subtropical rain forest of the study area.

Delta esuriens Fabricius, 1787 (Fig. 10a, b)

Material examined

Haryankot $1 \delta^{\lambda}(23 / 09 / 2017)$, Ghanidheri 2 ㅇ

(09/05/2018), $\quad 1+\quad(19 / 05 / 2018), \quad 1+$

$(01 / 06 / 2018), \quad 1$ 우 $\quad(11 / 06 / 2018), \quad 1$ 우

(12/06/2018), $\quad 1$ 우 $\quad(22 / 06 / 2018), \quad 1 \hat{0}$ 


\begin{abstract}
(01/07/2018), $\quad 1 \overbrace{}^{\Uparrow} \quad(04 / 07 / 2018), \quad 1$ ㅇ (14/07/2018), $\quad 1$ 우 $\quad(16 / 07 / 2018), \quad 1$ 우 (18/07/2018), 1 ㅇ, 1 ふ $(22 / 07 / 2018), \quad 1 \circlearrowleft$ (30/07/2018), $\quad 1$ 우 $\quad(01 / 08 / 2018), \quad 1$ ㅇ (04/08/2018), $\quad 1 \sigma^{\lambda} \quad(11 / 08 / 2018), \quad 1$ 的 (14/08/2018), $\quad 1 \hat{\jmath} \quad(17 / 08 / 2018), \quad 1 \hat{\jmath}$ (18/08/2018), $\quad 1$ 우 $\quad(19 / 08 / 2018), \quad 1$ q (30/08/2018), Wartair 5ㅇ, 3તे (14/05/2018), $2 \widehat{\delta}(09 / 06 / 2018)$, Petaw $2 \hat{\sigma}(02 / 09 / 2018)$.
\end{abstract}

\section{Distribution}

Arabia; China; Bhutan; Indonesia; Iran; Iraq; Israel; Laos; Mauritius; Myanmar; New Caledonia; Oman; Pakistan; Philippines; Qatar; Saudi Arabia; Thailand; Sri Lanka; United Arab Emirates; Vietnam [12, 15].

\section{Remarks}

Early reported from Khyber Pakhtunkhwa: Mansehra, Dir and Khanpur [18]; from Rawalpindi-Islamabad, Chakwal and Attock [13]; from Islamabad, Jummu \& Kashmir [17] and from Lower Dir [19]. Delta esuriens reported for the first time from subtropical rain forest of Malakand by present study.

Delta pyriforme pyriforme Fabricius, 1775 (Fig. 11)

\section{Material examined}

Ghanidheri1 ${ }^{\Uparrow}(21 / 09 / 2018)$.

\section{Distribution}

Bhutan; Cambodia; China (including Taiwan and Hong Kong); Hawaii; Indonesia; Laos; Malaysia; Moluccas; Myanmar; Nepal; New Guinea; Pakistan; Sri Lanka; Thailand; Vietnam [12, 15].

\section{Remarks}

Previously reported from Punjab, Peshawar and Islamabad [18]; from IslamabadRawalpindi [13]. For the first time present study reported Delta pyriforme pyriforme from subtropical rain forest of Malakand.

Allorhynchium metallicum de Saussure, 1852 (Fig. 12a, b)

\section{Material examined}

Ghanidheri 1q, đ̋1 (15/09/2017), 4 우 (12/05/2018), 1 ○ิ, 1 ㅇ $(15 / 05 / 2018), \quad 1{ }^{\lambda}$

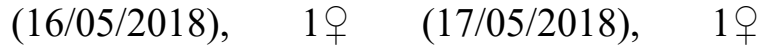
(06/06/2018), $1 \hat{\jmath}(21 / 06 / 2018), \quad 2$ ㅇ, $1 \hat{\jmath}$ (23/06/2018), $1 \hat{\jmath}(24 / 06 / 2018), 1+1$, $1 \hat{\jmath}$ $(27 / 06 / 2018), \quad 1$ 우 $\quad(28 / 06 / 2018), \quad 1$ 우 (30/06/2018), $\quad 1$ 우 $\quad(01 / 07 / 2018), \quad 2$ 우
(03/07/2018), $\quad 1$ 우 $(11 / 07 / 2018), \quad 1 \lesssim, \quad 1$ 우 $(21 / 07 / 2018), \quad 3 \quad(04 / 08 / 2018), \quad 2 \hat{\jmath}, \quad 1$ 우 (14/08/2018), $\quad 10^{\lambda} \quad(17 / 08 / 2018), \quad 1$ ㅇ (05/09/2018), Haryankot 1 ㅇ (23/09/2017), Dargai 2 + (03/07/2018), Khatakoshah 4, 4 $\overbrace{}^{\Uparrow}$ (19/08/2018), Meherdai $1+\quad(29 / 08 / 2018)$, Petaw 1 (02/09/2018).

\section{Distribution}

China; Bhutan; Indonesia; Laos; Malaysia; Myanmar; Nepal; Pakistan; Philippines; Singapore; Thailand $[4,13,15,22]$.

\section{Remarks}

Early reported from Chakwal, Attock, Jhelum, Rawalpindi and Islamabad [12]; from KhyberPakhtunkhwa: Chitral; Punjab: Changa Manga and Gilgit-Baltistan: Skardu [16]. Allorhynchium metallicum reported for the first time from subtropical rain forest of Malakand by this study.

Anterhynchium abdominale abdominale Illiger, 1802 (Fig. 13a, b)

Material examined

Khatakoshah $2 \hat{\jmath}, \quad 3 q \quad(19 / 08 / 2018)$,

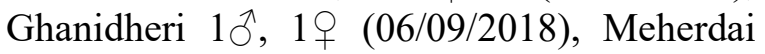
2 ㅇ (29/08/2018), Petaw 2 ㅇ (02/09/2018).

\section{Distribution}

Pakistan, India, Sri Lanka, China, Myanmar and Vietnam $[4,15]$.

\section{Remarks}

Early reported from Islamabad [17]. This study reported Anterhynchium abdominale abdominale for the first time from subtropical rain forest of Malakand.

Rhynchium oculatum leviscutis Cameron, 1908 (Fig. 14a, b)

Material examined

Ghanidheri $\quad 1 q \quad(25 / 05 / 2018), \quad 1 \delta$ (30/06/2018).

\section{Distribution}

India, Afghanistan $[4,15]$.

\section{Remarks}

Present study reported this species for the first time from Pakistan as there was no previous record of this species documented from the country.

Rhynchium carnaticum Fabricius, 1798 (Fig. $15 \mathrm{a}, \mathrm{b})$ 
Material examined

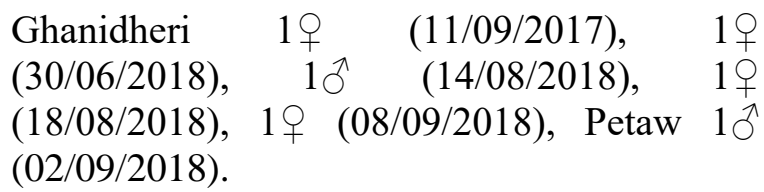

Distribution

Pakistan, India, Sri Lanka and Indonesia [15, 23].

\section{Remarks}

Early report from Swat [21]. Rhynchium carnaticum reported for the first time from subtropical rain forest of the area by present study.

Rhynchium brunneum brunneum Fabricius, 1793 (Fig. 16)

\section{Material examined}

Ghanidheri 1 우 $\quad(07 / 05 / 2018), \quad 1$ 우 (23/09/2018).

\section{Distribution}

Bangladesh; Bhutan; Cambodia; China (including Taiwan); Indonesia; Laos; Malaysia; Marianas; Myanmar; Nepal; New Britain; Pakistan; Palau; Seychelles; Thailand; Vietnam $[4,15]$.

\section{Remarks}

Early reported from Rawalpindi and Attock [13]. We reported Rhynchium brunneum brunneum for the first time from Khyber Pakhtunkhwa Province of Pakistan as there was no previous record of this species documented from this Province.

Eumenes punctatus de Saussure, 1852 (Fig. 17)

Material examined

Ghanidheri 1 우 (15/09/2017), 1 우 (29/09/2018), 1우 (30/09/2018), Haryankot 2 우 (22/09/2017).

\section{Distribution}

Pakistan, Russia, China, Sri Lanka, India, Japan, Korea and Russia [7, 15].

Remarks

Early report from Attock [13]. We reported Eumenes punctatus for the first time from Khyber Pakhtunkhwa Province of Pakistan as there was no previous record of this species documented from this Province.

Antepipona sibilans Cameron, 1903 (Fig. 18a, b)

\section{Material examined}

Haryankot 1 ㅇ (22/09/2018), Ghanidheri 1 ㅇ (22/08/2018), 1 우 (26/08/2018), Meherdai 1우, $1{ }^{\widehat{D}}(29 / 08 / 2018)$.

\section{Distribution}

Pakistan, Nepal and India [4].

Remarks

Early report from Attock, Rawalpindi, Islamabad and Chakwal [13]. Present study collected Antepipona sibilans for the first time from Khyber Pakhtunkhwa Province of Pakistan.

Antepipona deflenda Saunders, 1853 (Fig. 19) Material examined

Ghanidheri $\quad 1$ 우 $\quad(11 / 09 / 2017), \quad 2$ 우 $(25 / 08 / 2018)$.

Distribution

Pakistan, China, Iraq, Iran, Turkey, Uzbekistan, Albania, Lebanon, Austria, Russia, Germany, France, Portugal and Spain [24].

\section{Remarks}

Early report from Swat [21]. We reported Antepipona deflenda for the first time from subtropical rain forest of Malakand. 


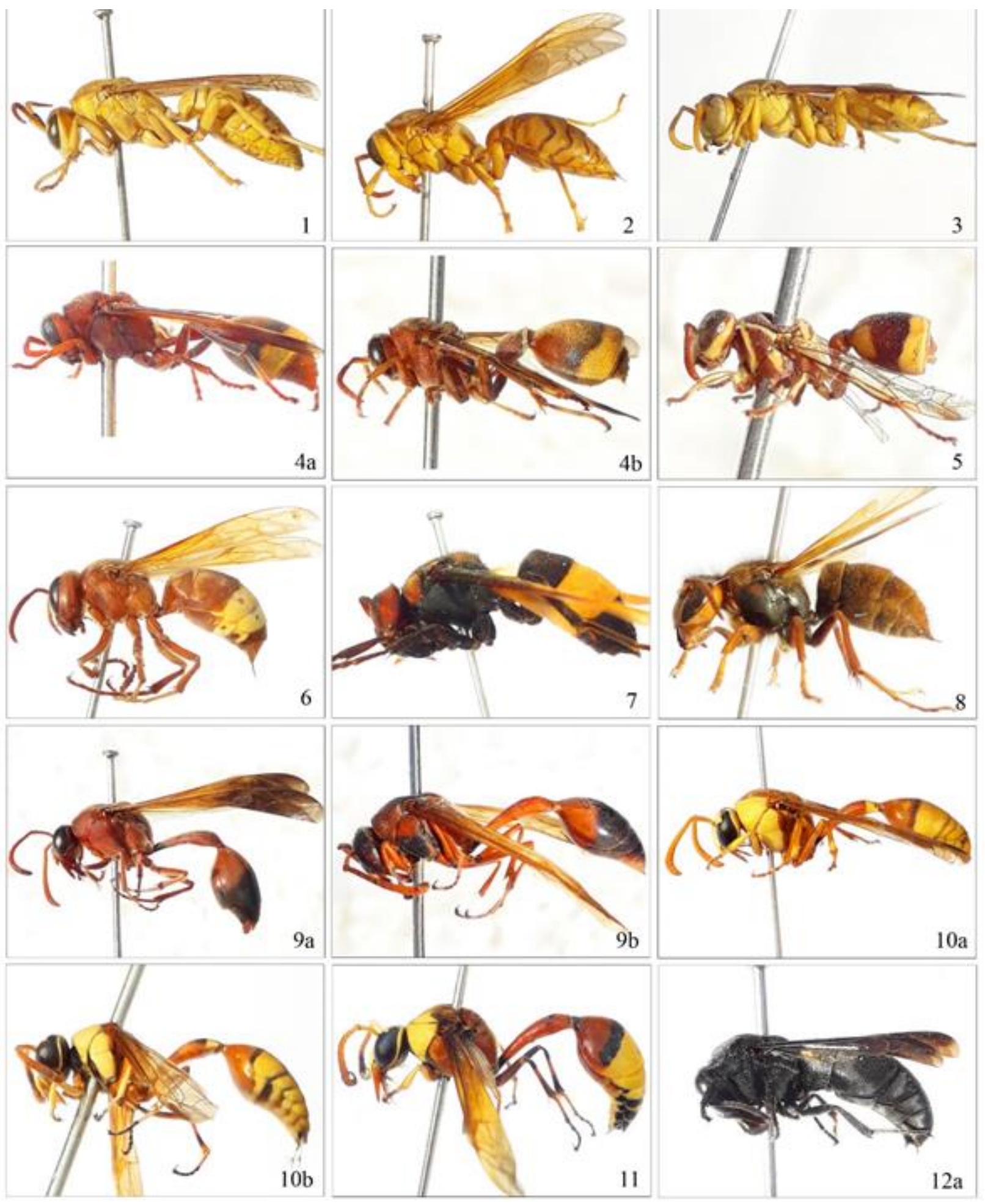

Figure (1-12a): 1. Polistes indicus $\odot$, 2. Polistes olivaceus ${ }_{+}$, 3. Polistes wattii + , 4a. Ropalidia brevita ${ }_{+}$, 4b. Ropalidia brevita $\bigcirc$, 5. Ropalidia cyathiformis $\odot$, 6. Vespa orientalis $\odot$ 7. Vespa

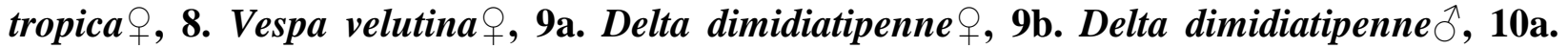
Delta esuriens $\bigcirc$, 10b. Delta esuriens ${ }^{\gamma}, 11$. Delta pyriforme pyriforme ${ }^{\circ}, 12 \mathrm{a}$. Allorhynchium metallicum ㅇ 


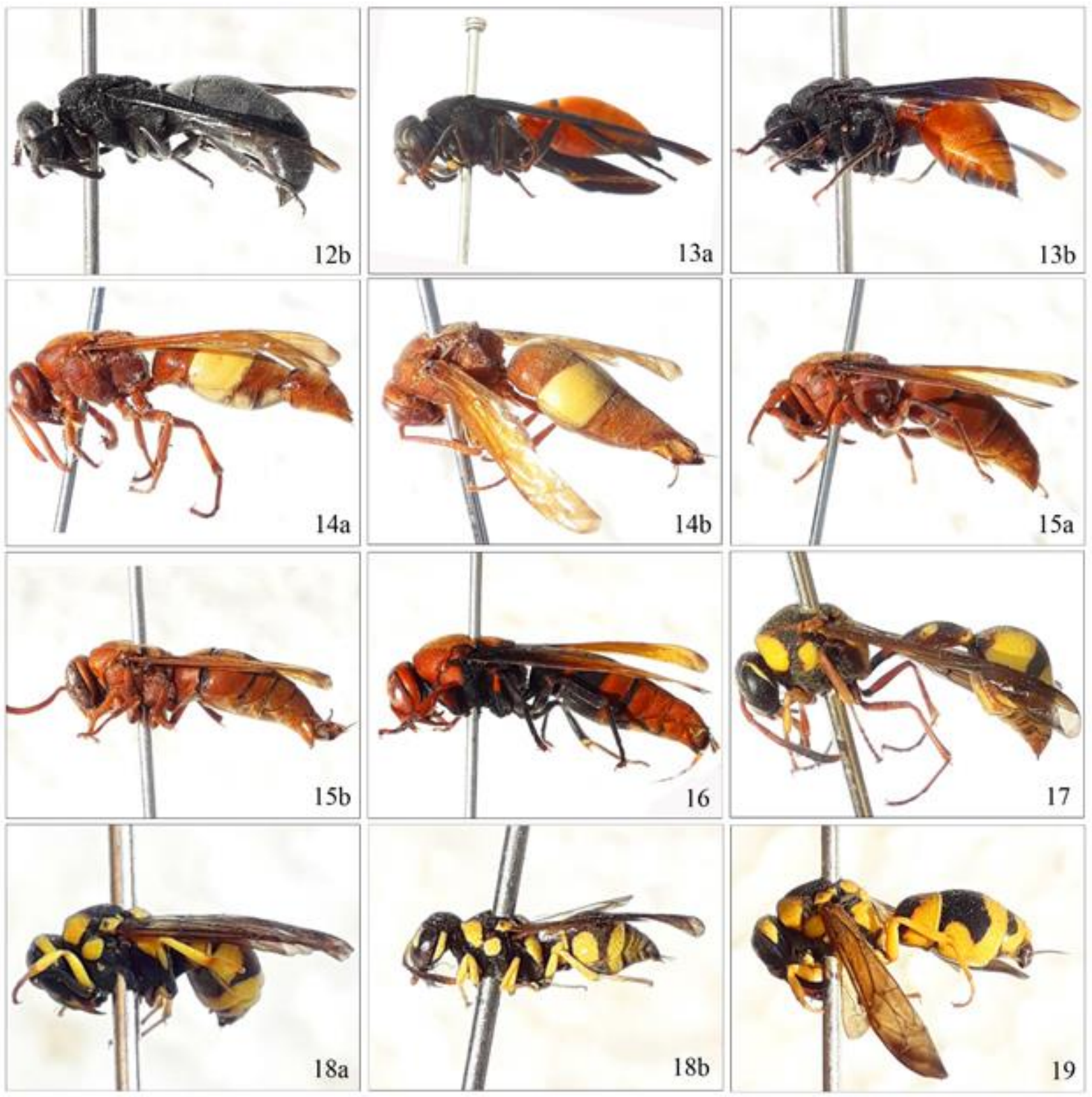

Figure (11b-19) 12b. Allorhynchium metallicum ${ }^{\wedge}$, 13a. Anterhynchium abdominale abdominale + , 13b. Anterhynchium abdominale abdominale $\curvearrowright$, 14a. Rhynchium oculatum leviscutis ${ }_{+}$, 14b. Rhynchium oculatum leviscutis ${ }^{\lambda}, 15 \mathrm{a}$. Rhynchium carnaticum + , $15 \mathrm{~b}$. Rhynchium carnaticum ${ }^{\lambda}$, 16. Rhynchium brunneum brunneum ${ }^{\circ}$, 17. Eumenes punctatus + ,

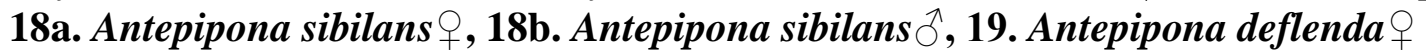

\section{Conclusion}

In conclusion, we have identified total 19 species of wasps from district Malakand, among which one Rhynchium oculatum leviscutis was $1^{\text {st }}$ record from Pakistan, 3 species: Rhynchium brunneum brunneum, Eumenes punctatus, Antepipona sibilans was $1^{\text {st }}$ record

from

Khyer Pakhtunkhwa, while 14 species: Polistes indicus, Polistes olivaceus, Polistes wattii, Ropalidia brevita, Delta dimidiatipenne, Delta esuriens, Delta pyriforme pyriforme, Ropalidia cyathiformis, Vespa orientalis, Vespa tropica, Vespa 
velutina, Allorhynchium metallicum, Anterhynchium abdominale abdominale, Rhynchium carnaticum, and Antepipona deflenda. were recorded from district Malakand for the first time

\section{Authors' contributions}

Conceived and designed the experiments: Q Khan \& Q Zaman, Performed the experiments: S Ahmad, Q Zaman, M Ismail, Ahmadullah, R Ali, U Ali \& Q Jamal, Analyzed the data: S Ahmad, Q Zaman \& Q Khan, Contributed materials/ analysis/ tools: Q Khan, Q Zaman \& G Rahman, Wrote the paper: Q Zaman, Q Khan, S Ahmad \& H Khan.

\section{Acknowledgement}

The authors are thankful to Dr Muhammad Rasool, Government College Kaqbal Swat, Khyber Pakhtunkghwa, for providing necessary help in finding the relevant literature.

\section{References}

1. Brusca RC \& Brusca GJ (2003). Invertebrates. Sinaur Associates, Sunderland, Massachusetts. ISBN 087893-097-3.

2. Richards OW \& Davies RG (1977). Imms' general textbook of Entomology 10th ed John Wiley \& Sons, New York.

3. Hoagstrom CW (2002). Magill's encyclopedia of science (Vol 1 Aardvarks-Endoskeletons) Salem Press, Inc. Pasadena, California, Hackensack, New Jersey.

4. Kumar PG \& Sharma G (2014). Taxonomic Studies on vespid wasps (Vespidae: Vespoidea: Hymenoptera: Insecta) of Rajasthan, India with Six New Records from the State. J on New Biol Rep 3(3): $240-258$

5. Moore GC \& Merchant ME (2010). Paper Wasps, Yellowjackets and Solitary Wasps. Texas A\&M AgriLife Extension Service, USA.

6. Buck M, Marshall SA \& Cheung DKB (2008). Identification atlas of the Vespidae (Hymenoptera, Aculeata) of the northeastern Nearctic region. Cana $J$ of Arthro Identification 5(1): 1-492.
7. Das BP \& Gupta VK (1989). The Social Wasps of India and the Adjacent Countries (Hymenoptera: Vespidae) The Association for the Study of Oriental Insects, PO Box 13148, Gainesville, Florida 32604-1148, USA.

8. Capinera JL (2008). Encyclopedia of Entomology 2nd ed, Vol 4 S-Z. Springer, Heidelberg. ISBN 978-1-4020-6359-6, ISBN 978-1-4020-6360-2.

9. Carpenter JM \& Nguyen LTP (2003). Keys to the genera of social wasps of South-East Asia (Hymenoptera: Vespidae). Entomol Sci 6: 183-192.

10. Dvorak L (2007). The social wasp fauna of Pakistan (Hymenoptera, Vespidae). Linzer Biol. Beitr 39(1): 51-55.

11. Kumar GP, Carpenter JM, Castro L \& Sureshan PM (2017). A taxonomic review of the Indian species of the genus Eumenes Latreille (Hymenoptera: Vespidae: Eumeninae) Zootaxa 4317(3): 469-498.

12. Faiz A, Rafi MA, Zia A, Shah A, Shah SW, Khan RU \& Saeed M (2016). Wasp fauna of (Eumeninae, Vespinae and Polistinae) in forests of Gilgit Baltistan (Pakistan). Pure and_Appl Biol 5(4): 682688.

13. Siddiqui JA, Bodlah I, Carpenter JM, Naeem M, Ahmad M, Bodlah MA (2015). Vespidae (Hymenoptera) of the Pothwar region of Punjab, Pakistan. Zootaxa 3914(5): 501-524.

14. Khan K, Rasool M \& Zahid M (2018). Taxonomic study of polistinae species (Hymenoptera: Vespidae) of Dir, Pakistan. $J$ of Entomol and Zool Stu 6(2): 791-794.

15. Carpenter JM, Kumar G, Gawas S (2020). An annotated distributional checklist of Vespidae (Hymenoptera: Vespoidea) of India. Zootaxa 4784(1): 01-87.

16. Barthelemy CA (2008). Provisional identification guide to the social vespids of Hong Kong (Hymenoptera: Vespidae), Centre for Land and Biological Resources Research, Agriculture Canada, pp. 2-132.

17. Rafi MA, Carpenter JM, Qasim M, Shehzad A, Zia A, Khan MR, Mastoi MI, 
Naz F, Ilyas M, Shah M \& Bhatti AR (2017). The vespid fauna of Pakistan. Zootaxa 4362(1): 001-028.

18. Inayatullah M (2012). To the knowledge of Vespidae (Hymenoptera) of Pakistan. Zootaxa 3318: 26-50.

19. Rauf SA, Rafi MA, Qasim M, Sheikh K, Mehmood K, Durrani S, Khan A \& Rahman A (2018). Wasp fauna of subfamilies (Eumeninae, Polistinae, Vespinae) from Dir, Khyber Pakhtunkhwa, Pakistan. Uni of Sindh $J$ of Ani Sci 2(1): 1-7.

20. Carpenter JM \& Genaro JA (2011). Vespidae (Insecta: Hymenoptera) of Puerto Rico, West Indies. Insecta Mundi. Paper 714.

21. Rasool M, Zahid M, Khan Q, Khan K \& Ismail M (2017). Vespinae Wasps (Vespidae: Hymenoptera) of Swat Khyber
Pakhtunkhwa Pakistan. Wulfenia J, Klagenfurt, Australia ISSN 1561-882X.

22. Dorji P, Klein W \& Nidup T (2016). Study of Paper wasps (Hymenoptera: Vespidae: Polistinae) of Bhutan. Bio Bulletin, Honorary Research Associate, Naturalis Biodiversity Centre, 2300 RA Leiden, Netherlands. 2(1): 128-136.

23. Pannure A, Belavadi VV \& Carpenter JM (2016). Taxonomic studies on potter wasps (Hymenoptera: Vespidae: Eumeninae) of south India. Zootaxa 4171(1): 1-50.

24. Khan MT, Rafi MA, Sultana R, Munir A $\&$ Ahmad S (2018). Wasps of subfamilies Eumeninae, Vespinae and Polistinae from Sindh-Pakistan. $J$ of Entomol and Zool Stu 6(2): 